\title{
A Study of the Extragalactic UV Radiation in Helix Nebula using GALEX
}

\section{Lakshmi S BOSE} Department of Physics, Faculty of Sree Narayana Mangalam College, Maliankara, Ernakulam,
Kerala 693516, India

\author{
(Corresponding author's e-mail: lakshmisbose@gmail.com)
}

Received: 10 April 2021, Revised: 14 May 2021, Accepted: 21 May 2021

\begin{abstract}
We have studied the ultraviolet sources using Galaxy Evolution Explorer medium imaging surveys in Helix Nebula and estimated UV fluxes by using aperture photometry in distant and near ultraviolet bands. The aperture photometric method produces reliable, accurate flux measurements and found inconsistent with the merged catalog of Galaxy Evolution Explorer. From the current results, the fluxes are consistent with brighter absolute magnitude up to 24.5 and the measurement error increases gradually to more than $50 \%$ at the fainter magnitude side. Percentage of error in far UV is greater than near UV, due to the fact that brighter galaxies are more visible than the near UV sources. The diffuse UV contributors of zodiacal light, airglow contribution in the nebula were estimated. The total extragalactic UV radiation from the detected sources to the diffuse background in the nebula is of the order of $50 \pm 14$ photons $\mathrm{cm}^{-2} \mathrm{sr}^{-1} \mathrm{~s}^{-1} \AA^{-1}$ in NUV band and $28 \pm 10$ photons $\mathrm{cm}^{-2} \mathrm{sr}^{-1} \mathrm{~s}^{-1} \AA^{-1}$ in FUV band.
\end{abstract}

Keywords: Diffuse UV, Extragalactic UV radiation, FUV-NUV fluxes, GALEX observations

\section{Introduction}

One of the largest spectacular-known planetary nebulae, the Helix Nebula (NGC 7293) in the constellation of Aquarius, about 650 light years away from the Earth, is clearly recognizable in the ultraviolet sky (UV) [1]. The nebula is largely composed of molecular gas, ionized material and interstellar dust. The Helix's array of thousands of filamentary structures, or gas threads is a striking feature that was first discovered by ground-based images [2]. The region was extensively studied using the European Southern Observatory's Very Large Telescope (ESO's VLT) and Hubble Space telescope but was not completely understood. The spatial structure of helix nebula was studied by Meixner et al. [6] on the characterized atomic gas in this region using Hubble Space Telescope [3-6]. Morphological study of helix nebula in the infrared region helps to understand that the nebula has a central star and shows high density with cometary knots [7,8]. Recently, a white dwarf has been detected at the nebula's center with intense UV radiation that heats the gas expelled layers which shine brilliantly in the infrared (IR). Spitzer, IR mission captured the precise infrared signature of the dust and gas, while Galaxy Evolution Explorer $(G A L E X)$ UV mission picked out the ultraviolet light pouring out of this system. Herschel observations in the helix nebula were used to explain the dust and energy distributions over the region [9]. Molecular hydrogen $\left(\mathrm{H}_{2}\right)$, $\mathrm{CO}$ at infrared in a large number of globules of helix was observed and complex molecules like $\mathrm{CN}, \mathrm{HCO}+, \mathrm{HCN}$ have been identified in the nebula [10-14]. The spatial distribution of the atomic and molecular gas with the SPIRE instrument was studied by Etxaluze et al. [15].

Many astronomical missions are available in ultraviolet wavelength, but Galaxy Evolution Explorer $-50 \mathrm{~cm}$ telescope conducted the first all sky surveys in UV [16]. Ultraviolet imaging of planetary nebulae with GALEX was taken to illustrate the variety in UV morphology [17]. The current study that used the observations of GALEX in helix planetary nebula can explain the nature of ultraviolet emission 
http://wjst.wu.ac.th

and the UV flux distribution of extragalactic sources. However, the region is not fully exposed to source detection and diffuse radiation studies in the ultraviolet.

\section{Observations}

The detailed description, performance, and data characterization of Galaxy Evolution Explorer (GALEX) was given by Martin et al. [16], Morrissey et al. [18] and Bianchi [19]. The 2 imaging surveys of the satellite in UV band: 135 - 180 and 180 - $200 \mathrm{~nm}$ has a significant spatial resolution of 3"- 6" with $1.28^{\circ}$ field of view (FOV). The sky images contain UV sources and diffuse background sources were produced by GALEX pipeline. The latest science-enhanced catalogs of UV sources are available for GALEX observations [20].

We have exerted an effort towards source detection in ultraviolet using GALEX observations. A set of 5 GALEX medium imaging surveys of Helix Nebula centered at $22 \mathrm{~h} 29^{\mathrm{m}} 38^{\mathrm{s}}, 20^{\circ} 29^{\prime} 12^{\prime \prime}$ within 5 square degrees were studied. The observational details of GALEX such as tile center in equatorial coordinate system, exposure time is of the order of $1000 \mathrm{~s}$ and visits in two UV bands is tabulated (Table 1).

Table 1 Observation Log of GALEX medium imaging surveys in Helix Nebula.

\begin{tabular}{ccccccc}
\hline Tiles name & $\begin{array}{c}\text { Right } \\
\text { ascension } \\
\text { (degree) }\end{array}$ & $\begin{array}{c}\text { Declination } \\
\text { (degree) }\end{array}$ & $\begin{array}{c}\text { NUV } \\
\text { exposure time } \\
\text { (seconds) }\end{array}$ & $\begin{array}{c}\text { FUV } \\
\text { exposure time } \\
\text { (seconds) }\end{array}$ & $\begin{array}{c}\text { NUV } \\
\text { visits }\end{array}$ & $\begin{array}{c}\text { FUV } \\
\text { visits }\end{array}$ \\
\hline MIS2DFSGP_30441_0008 & 338.12 & -23.41 & 3115 & 711 & 2 & 1 \\
MIS2DFSGP_30502_0066 & 338.70 & -24.40 & 1543 & 1543 & 1 & 1 \\
MIS2DFSGP_30567_0064 & 336.05 & -24.85 & 2350 & 726 & 2 & 1 \\
MIS2DFSGP_30508_0005 & 332.37 & -23.23 & 176 & 176 & 1 & 1 \\
MIS2DFSGP_30568_0063 & 334.97 & -24.66 & 1600 & 560 & 2 & 1 \\
\hline
\end{tabular}

\section{Methodology}

GALEX photometric catalog produced by SExtractor, extracted the flux details in counts per pixel for all UV sources in the Helix nebula. We have obtained about 48,464 sources (stars - 23,761; galaxies 24,703 ) and their flux details in this region from the merged catalog. Identification of UV-stars, galaxies from the GALEX merged source catalog is based on the stellarity index (CLASS_STAR) value following Frayer et al. [21] and Groenewegen et al. [22] as given below.

Object to be consider as star,

\section{IF CLASS STAR $>$ 0:85 for $R<23$; \\ CLASS STAR $>$ 0:90 otherwise:}

Here the flux measurements in two UV bands are conducted using the aperture photometry technique for all the detected sources in the absolute magnitude (AB magnitude) 17 - 30 range [23].

GALEX merged catalog has sources only within the range of $\mathrm{AB}$ magnitude 17 - 24.5 (Figure 1). The number of UV sources decrease at the fainter magnitude side. The reason may be due to the low resolution of GALEX, estimation error of UV background by SExtractor and source confusion (Overlapping fainter sources). Hence, source center positions are marked in the GALEX MIS intensity images using its merged catalog data and conducted aperture photometry for all detected sources in FUV 
and NUV bands. In this study, we adopted the technique to detect fainter UV sources and flux measurements by eliminating the sky background and make a fixed radius of the inner annulus as 7.0 arcsec and outer annulus as 10.0 arcsec from the center of each source (Figure 2). Aperture slice shows flux variation of the detected sources in the GALEX catalog. Where, NUV fluxes give accurate value for the selected source whose center has the maximum peak value and gradually decreases towards outer aperture, and makes a good Gaussian profile, despite FUV flux spreads throughout the region (Figure 3 and 4).



Figure 1 Number of UV sources detected using SExtractor in the GALEX merged catalog.

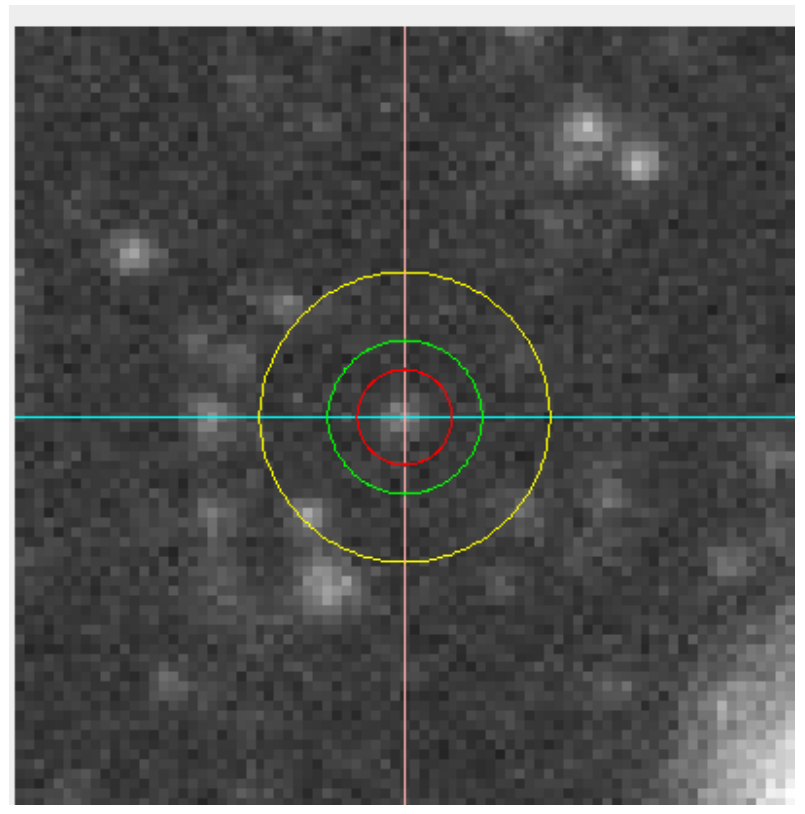

Figure 2 Flux measurements by photometric method with inner annulus \& outer annulus as 7.0" (red and green) and 10.0" (yellow), respectively. 


\section{Aperture Slice}

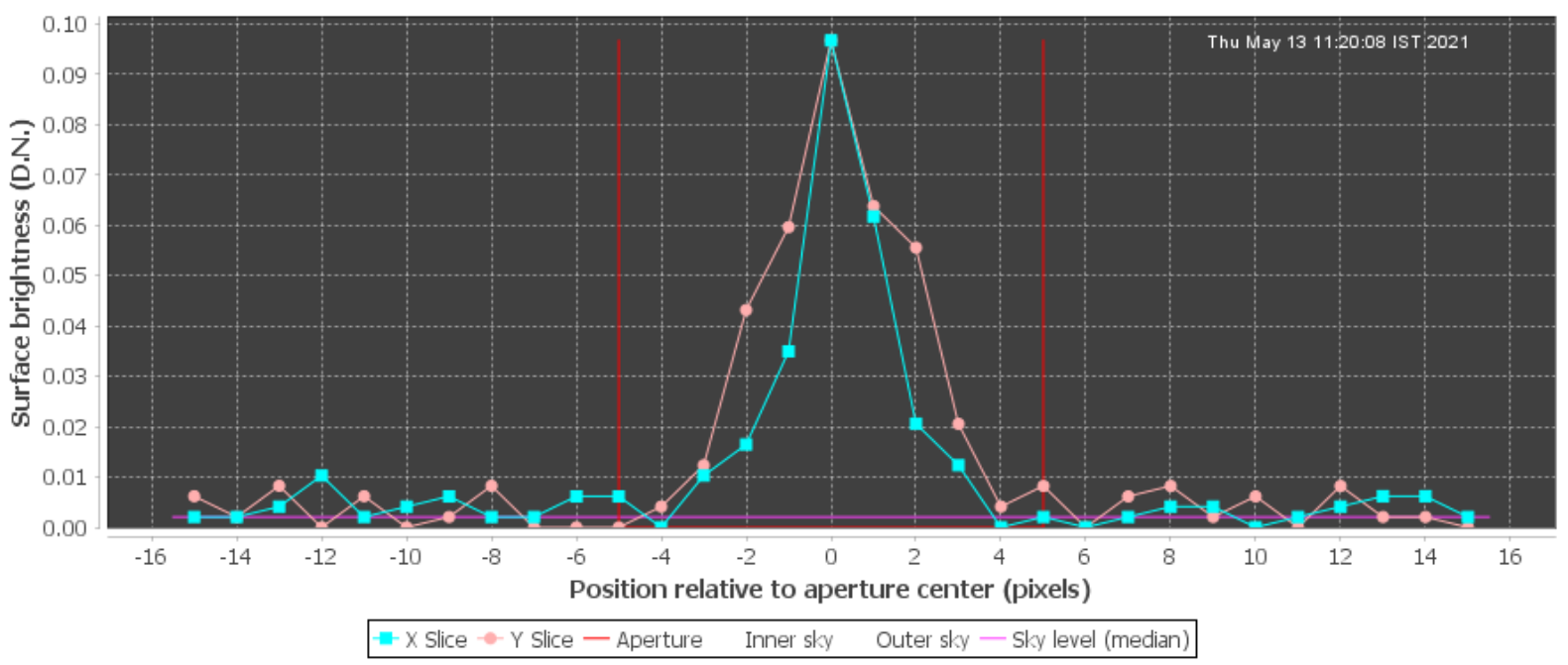

Figure 3 NUV- flux variations in count per pixel for the GALEX detected source within 5 radiuses (red) $\&$ peak shows the source center has maximum surface brightness.

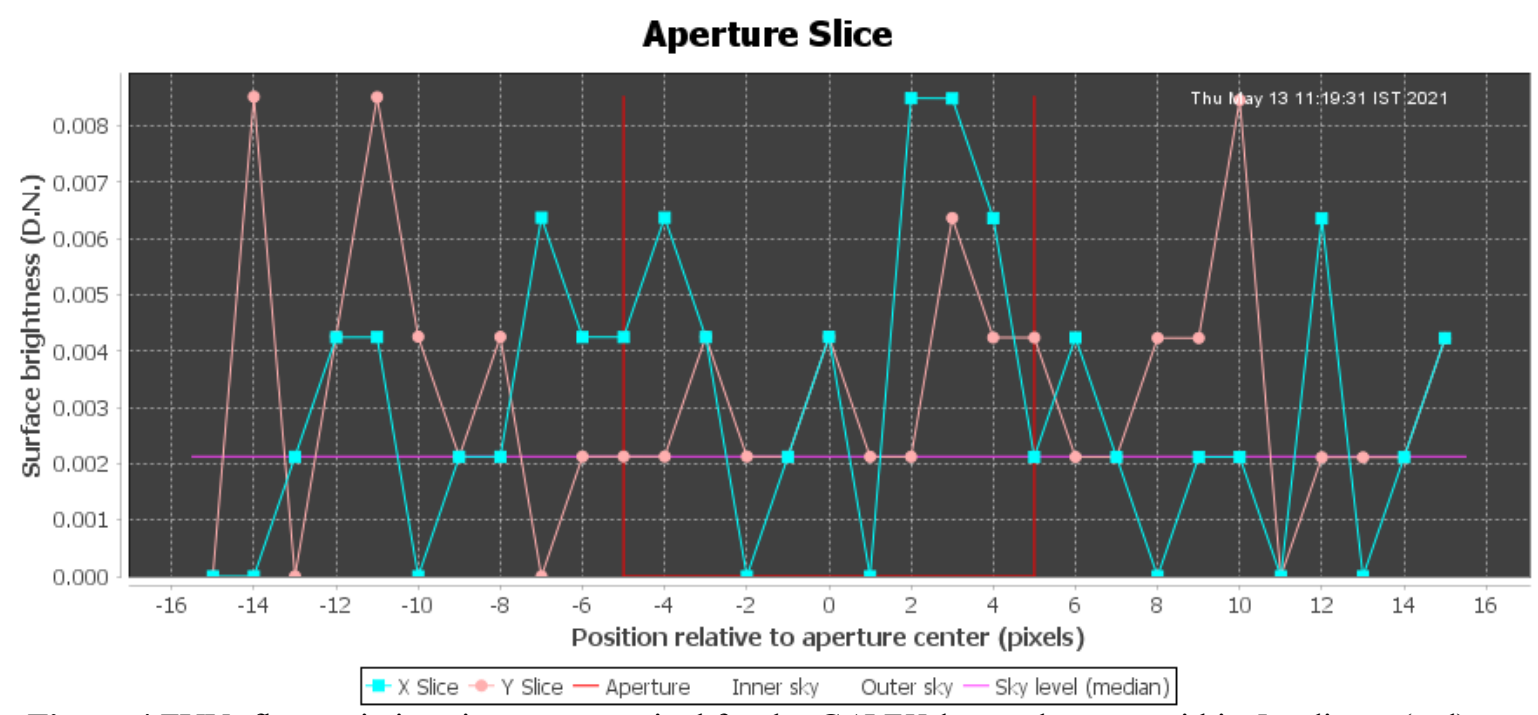

Figure 4 FUV- flux variations in count per pixel for the GALEX detected source within 5 radiuses (red).

Many studies were conducted for the estimation of diffuse UV radiation; emission other than point sources in the past, but limited with instrumental sensitivity [24,25]. To study the diffuse UV radiation, adopt a method in binning images into $100 \times 100$ GALEX pixels in size, estimate background radiation and eliminate all the edge effects by taking 1.15/1.28 ${ }^{\circ}$ FOV [26,27]. The characterization of foreground emission Airglow and zodiacal light in each field is done by the optical distribution and Telemetered Event Counter (TEC) respectively (Table 2) [28]. Optical depth in this region is $0.09-0.05$ with E (B-V) variation is 0.01 to 0.04 magnitudes [29]. 
Table 2 Airglow and zodiacal light contribution in photons $\mathrm{cm}^{-2} \mathrm{sr}^{-1} \mathrm{~s}^{-1} \AA^{-1}$ units for each field.

\begin{tabular}{ccccc}
\hline \multirow{2}{*}{ Tiles name } & \multicolumn{2}{c}{$\begin{array}{c}\text { Average } \\
\text { airglow }\end{array}$} & \multicolumn{2}{c}{$\begin{array}{c}\text { Average } \\
\text { zodiacal light }\end{array}$} \\
\cline { 2 - 5 } & NUV & FUV & NUV & FUV \\
\hline MIS2DFSGP_30441_0008 & 335 & 325 & 482 & 485 \\
MIS2DFSGP_30502_0066 & 321 & 334 & 481 & 479 \\
MIS2DFSGP_30567_0064 & 326 & 322 & 487 & 479 \\
MIS2DFSGP_30508_0005 & 336 & 333 & 488 & 481 \\
MIS2DFSGP_30568_0063 & 331 & 330 & 484 & 483 \\
\hline
\end{tabular}

\section{Results and discussions}

The duplicate sources in this field due to the overlapping of GALEX observations are identified and carefully removed. There is a drop out of galaxies at AB magnitude 24.5 in GALEX merged catalog, this may be due to the overestimation of sky background of GALEX source SExtractor (Figure 1). On the other hand, a total 20,203 UV sources are detected at the fainter magnitude side 25.0 - 28.0 using aperture photometry and measured UV fluxes by aperture photometric method, which are not included in the GALEX source list. The number counts of galaxies and $\mathrm{AB}$ magnitudes in Helix nebula within $0.8^{\circ}$ is calculated using aperture photometry (Table 3). The GALEX can easily detect brighter sources up to the $\mathrm{AB}$ magnitude of 22.0. Due to the low resolution, two nearby fainter sources in the intensity map with average sky background subtraction SExtractor may turn out as a brighter source were included in the catalog list. There are about 6,322 such sources that are found in this region. The sensitivity of the results is checked by inserting sources artificially at random positions on images at different magnitudes. The SExtractor gets the same results as in the catalog, while the photometric method could find more sources at fainter magnitude up to the absolute magnitude of 28.0.

Table 3 Number of extragalactic sources in UV field by photometric method in Helix Nebula in AB magnitude 17 to 28 range.

\begin{tabular}{ccccc}
\hline AB Mag & $\mathbf{N}_{\text {NUVobjects }}$ & Log $\left(\mathbf{N}_{\text {NUVobjects }} / \mathbf{d e g}^{\mathbf{2}} / \mathbf{m a g}\right)$ & $\mathbf{N}_{\text {FUVobjects }}$ & Log $\left(\mathbf{N}_{\text {FUVobjects }} / \mathbf{d e g}^{\mathbf{2}} / \mathbf{m a g}\right)$ \\
\hline 17.0 & 2 & 0.32 & 1 & 0.02 \\
18.0 & 4 & 0.62 & 3 & 0.49 \\
19.0 & 38 & 1.6 & 32 & 1.52 \\
20.0 & 95 & 2.0 & 90 & 1.97 \\
21.0 & 1382 & 3.16 & 557 & 2.76 \\
22.0 & 2291 & 3.38 & 914 & 2.98 \\
23.0 & 3627 & 3.58 & 1348 & 3.15 \\
24.0 & 5868 & 3.79 & 1901 & 3.30 \\
25.0 & 7869 & 3.91 & 2323 & 3.38 \\
26.0 & 9294 & 3.98 & 5108 & 3.73 \\
27.0 & 12452 & 4.11 & 8939 & 3.97 \\
28.0 & 14254 & 4.17 & 11918 & 4.09 \\
\hline
\end{tabular}


http://wjst.wu.ac.th

The sources detected both in aperture photometry and GALEX SExtractor are compared with their fluxes in FUV and NUV bands. The calculated errors in different AB magnitude for 5 GALEX observations are tabulated (Table 4). Comparing the GALEX merged catalog fluxes with photometric results for all such sources; the flux measurement errors are found to be less for brighter sources having $\mathrm{AB}$ magnitude up to 22.0 and increase gradually to more than $50 \%$ for fainter sources. The percentage of error in FUV is greater than NUV, this may be due to brighter galaxies are more visible in NUV than FUV.

Table 4 Percentage of error in FUV and NUV for 5 GALEX observations in 17 - 28 magnitude range.

\begin{tabular}{|c|c|c|c|c|c|}
\hline Tiles name & $\begin{array}{c}\text { Right } \\
\text { ascension } \\
\text { (degree) }\end{array}$ & $\begin{array}{c}\text { Declination } \\
\text { (degree) }\end{array}$ & $\begin{array}{l}\text { AB magnitude } \\
\text { range }\end{array}$ & $\begin{array}{l}\% \text { of error } \\
\text { variation in } \\
\text { FUV band }\end{array}$ & $\begin{array}{l}\% \text { of error } \\
\text { variation in } \\
\text { NUV band }\end{array}$ \\
\hline \multirow{11}{*}{ MIS2DFSGP_30441_0008 } & \multirow{11}{*}{338.12} & \multirow{11}{*}{-23.41} & $17.0-18.0$ & $0.5-1.4$ & $0.5-0.9$ \\
\hline & & & $18.0-19.0$ & $0.7-1.0$ & $0.7-1.0$ \\
\hline & & & $19.0-20.0$ & $0.9-1.0$ & $0.9-1.0$ \\
\hline & & & $20.0-21.0$ & $2.5-5.0$ & $2.2-3.0$ \\
\hline & & & $21.0-22.0$ & $11.5-23.0$ & $5.5-16.0$ \\
\hline & & & $22.0-23.0$ & $14.0-42.6$ & $17.0-22.9$ \\
\hline & & & $23.0-24.0$ & $21.0-45.5$ & 21.3. -25.5 \\
\hline & & & $24.0-25.0$ & $27.0-46.0$ & $27.0-32.0$ \\
\hline & & & $25.0-26.0$ & $31.0-53.8$ & $32.8-39.8$ \\
\hline & & & $26.0-27.0$ & $31.5-54.0$ & $32.9-40.5$ \\
\hline & & & $27.0-28.0$ & $32.3-54.4$ & $33.2-40.7$ \\
\hline \multirow{11}{*}{ MIS2DFSGP_30502_0066 } & \multirow{11}{*}{338.70} & \multirow{11}{*}{-24.40} & $17.0-18.0$ & $0.1-0.8$ & $0.1-0.5$ \\
\hline & & & $18.0-19.0$ & $0.7-1.0$ & $0.5-1.1$ \\
\hline & & & $19.0-20.0$ & $0.9-1.7$ & $0.6-1.1$ \\
\hline & & & $20.0-21.0$ & $2.5-5.0$ & $2.2-3.0$ \\
\hline & & & $21.0-22.0$ & $11.5-23.0$ & $7.8-18.3$ \\
\hline & & & $22.0-23.0$ & $16.0-29.6$ & $17.0-22.9$ \\
\hline & & & $23.0-24.0$ & $21.0-35.3$ & $21.3-26.7$ \\
\hline & & & $24.0-25.0$ & $25.0-40.0$ & $27.0-32.0$ \\
\hline & & & $25.0-26.0$ & $34.6-49.8$ & $28.8-37.8$ \\
\hline & & & $26.0-27.0$ & $34.8-49.9$ & $28.9-37.9$ \\
\hline & & & $27.0-28.0$ & $35.0-50.1$ & $29.3-38.2$ \\
\hline \multirow{11}{*}{ MIS2DFSGP_30567_0064 } & \multirow{11}{*}{336.05} & \multirow{11}{*}{-24.85} & $17.0-18.0$ & $0.5-1.4$ & $0.5-0.9$ \\
\hline & & & $18.0-19.0$ & $0.7-1.0$ & $0.7-1.2$ \\
\hline & & & $19.0-20.0$ & $0.6-1.0$ & $0.9-1.2$ \\
\hline & & & $20.0-21.0$ & $2.5-5.0$ & $2.2-3.0$ \\
\hline & & & $21.0-22.0$ & $11.5-23.0$ & $5.5-16.0$ \\
\hline & & & $22.0-23.0$ & $14.0-42.6$ & $17.0-22.9$ \\
\hline & & & $23.0-24.0$ & $21.0-45.5$ & 21.3. -25.5 \\
\hline & & & $24.0-25.0$ & $27.0-46.0$ & $27.0-32.0$ \\
\hline & & & $25.0-26.0$ & $31.0-53.8$ & $32.8-39.8$ \\
\hline & & & $26.0-27.0$ & $31.4-54.1$ & $32.9-40.1$ \\
\hline & & & $27.0-28.0$ & $31.9-54.9$ & $40.0-40.5$ \\
\hline \multirow{8}{*}{ MIS2DFSGP_30508_0005 } & \multirow{8}{*}{332.37} & \multirow{8}{*}{-23.23} & $17.0-18.0$ & $0.2-0.5$ & $0.5-0.9$ \\
\hline & & & $18.0-19.0$ & $0.7-1.0$ & $0.7-1.2$ \\
\hline & & & $19.0-20.0$ & $0.8-1.0$ & $0.9-1.4$ \\
\hline & & & $20.0-21.0$ & $3.5-5.0$ & $1.8-2.9$ \\
\hline & & & $21.0-22.0$ & $11.5-21.0$ & $7.5-9.0$ \\
\hline & & & $22.0-23.0$ & $19.7-27.6$ & $9.0-10.9$ \\
\hline & & & $23.0-24.0$ & $29.0-35.5$ & 11.3. -15.5 \\
\hline & & & $24.0-25.0$ & $37.0-44.0$ & $19.1-21.0$ \\
\hline
\end{tabular}




\begin{tabular}{cccccc}
\hline Tiles name & $\begin{array}{c}\text { Right } \\
\text { ascension } \\
\text { (degree) }\end{array}$ & $\begin{array}{c}\text { Declination } \\
\text { (degree) }\end{array}$ & $\begin{array}{c}\text { AB magnitude } \\
\text { range }\end{array}$ & $\begin{array}{c}\text { \% of error } \\
\text { variation in } \\
\text { FUV band }\end{array}$ & $\begin{array}{c}\text { \% of error } \\
\text { variation in } \\
\text { NUV band }\end{array}$ \\
\hline & & $25.0-26.0$ & $31.0-51.8$ & $22.8-35.8$ \\
& & $26.0-27.0$ & $31.5-52.0$ & $22.9-36.1$ \\
& & $27.0-28.0$ & $31.9-52.4$ & $23.3-36.6$ \\
& & $17.0-18.0$ & $0.3-1.7$ & $0.25-1.0$ \\
& & $18.0-19.0$ & $0.4-1.0$ & $0.9-1.0$ \\
& & $19.0-20.0$ & $0.9-1.0$ & $0.8-1.0$ \\
& & $20.0-21.0$ & $2.5-4.5$ & $1.2-2.8$ \\
& & $21.0-22.0$ & $9.5-21.9$ & $5.5-18.0$ \\
& & & $22.0-23.0$ & $22.0-40.6$ & $17.0-22.9$ \\
& & & $23.0-24.0$ & $21.0-45.5$ & $21.3-25.5$ \\
& & & $24.0-25.0$ & $27.0-46.0$ & $27.0-32.0$ \\
& & & $25.0-26.0$ & $31.0-50.8$ & $32.8-35.8$ \\
& & & $26.0-27.0$ & $31.4-51.4$ & $33.0-36.0$ \\
& & & $27.0-28.0$ & $31.8-51.9$ & $33.5-36.3$ \\
\hline
\end{tabular}

\section{Estimation of diffuse UV emission}

Zodiacal light, the contributor to diffuse NUV which depend upon the angle from the sun and the distance from the ecliptic plane. The contribution using the optical distribution by assuming that the ratio between the zodiacal light and the solar spectrum is the same at all wavelengths and found to be 485 photons $\mathrm{cm}^{-2} \mathrm{sr}^{-1} \mathrm{~s}^{-1} \AA^{-1}$ following the procedure of Bose et al. [30].

Airglow depends on solar activity having no consistent observations and we have calculated it as 330 photons $\mathrm{cm}^{-2} \mathrm{sr}^{-1} \mathrm{~s}^{-1} \AA^{-1}$ by TEC of the spacecraft to track the total number of counts as a function of orbital time. Total foreground emission for entire fields are obtained as 420 photons $\mathrm{cm}^{-2} \mathrm{sr}^{-1} \mathrm{~s}^{-1} \AA^{-1}$ with 5 photons $\mathrm{cm}^{-2} \mathrm{sr}^{-1} \mathrm{~s}^{-1} \AA^{-1}$ as GALEX instrumental dark count. After subtracting the foreground emission, the total extragalactic UV radiation from the detected sources to the diffuse background in the nebula is of the order of $50 \pm 14$ photons $\mathrm{cm}^{-2} \mathrm{sr}^{-1} \mathrm{~s}^{-1} \AA^{-1}$ in NUV band and $28 \pm 10$ photons $\mathrm{cm}^{-2} \mathrm{sr}^{-1} \mathrm{~s}^{-1} \AA^{-1}$ in the FUV band.

\section{Conclusions}

We have analyzed Helix Nebula using GALEX image surveys and found that our novel method to detect UV sources is more reliable and sensitive on the results at the fainter magnitude side. Photometric method can be used on a wide-scale, thereby solving problems related to GALEX SExtractor measurements. These investigations can extend to other UV observations to detect fainter sources. Moreover, this method is useful to study galactic and extragalactic contribution at low-mid-high galactic latitude regions of the sky. Compare with IR mission surveys, a multi wavelength studies of source detection will contribute towards the physical and chemical properties of galaxies and their evolution. The study of the number counts of UV sources can be used to measure the total luminosity of galaxies, testing of galaxy models and an indirect way to study Spectral Energy Distribution (SED) of galaxies. Small scale study of diffuse radiation in this region can be used for further modeling of dust scattered radiation. The Astrosat Sky surveys in UV band data, a multi wavelength astronomy mission can be used in the future to make a comparative study with these results for the estimation of the total extragalactic contribution which ultimately map the star formation history of the current universe.

\section{Acknowledgments}

This work is based on the data from NASA's GALEX GR6/GR7 program. We acknowledge the use of NASA's SkyView facility \& NASA's Astrophysics Data System. 


\section{References}

[1] L Bianchi. GALEX and star formation. Astrophys. Space Sci. 2011; 335, 51-60.

[2] R Cowen. On the trail of dead planets: Dust ring around a white dwarf. Sci. News 2007; 100, 171.

[3] CR O'Dell and WJ Henney. A multi-instrument study of the Helix Nebula knots with the Hubble Space Telescope. Astrophys. J. 2005; 130, 172-87.

[4] PAMV Hoof, GCVD Steene, KM Exter, MJ Barlow, T Ueta, MAT Groenewegen, WK Gear, HL Gomez, PC Hargrave, RJ Ivison, SJ Leeks, TL Lim, G Olofsson, ET Polehampton, BM Swinyard, HV Winckel, C Waelkens and R Wesson. A Herschel study of NGC 650. Astron. Astrophys. 2013; 560, A7.

[5] GF Benedict, BE McArthur, R Napiwotzki, TE Harrison, HC Harris, ENelan, HE Bond, RJ Patterson and R Ciardullo. Astrometry with the Hubble Space Telescope: Trigonometric parallaxes of planetary Nebula nuclei NGC 6853, NGC 7293, ABELL 31, and DeHt5. Astron. J. 2009; 138, 1969-84

[6] M Meixner, P McCullough, J Hartman, M Son and AK Speck. Molecular hydrogen knots in the Helix Nebula. Astron. J. 2005; 130, 1784-94.

[7] PAMV Hoof, GCVD Steene, MJ Barlow, KM Exter, B Sibthorpe, T Ueta, V Peris, MAT Groenewegen, JADL Blommaert, M Cohen, WD Meester, GJ Ferland, WK Gear, HL Gomez, PC Hargrave, E Huygen, RJ Ivison, C Jean, SJ Leeks, TL Lim, G Olofsson, ET Polehampton, S Regibo, P Royer, BM Swinyard, B Vandenbussche, HV Winckel, C Waelkens, HJ Walker and R Wesson. Herschel images of NGC 6720: $\mathrm{H}_{2}$ formation of dust grains. Astron. Astrophys. 2010; 518, L137.

[8] K Young, P Cox, PJ Huggins, T Forveille and R Bachiller. The molecular envelope of the Helix Nebula. Astrophys. J. 1999; 522, 387.

[9] GCVD Steene, PAMV Hoof, KM Exter, MJ Barlow, J Cernicharo, M Etxaluze, WK Gear, JR Goicoechea, HL Gomez, MAT Groenewegen, PC Hargrave, RJ Ivison, SJ Leeks, TL Lim, M Matsuura, G Olofsson, ET Polehampton, BM Swinyard, T Ueta, HV Winckel, C Waelkens and R Wesson. Herschel imaging of the dust in the Helix Nebula (NGC 7293). Astron. Astrophys. 2015; 574, A134

[10] AK Speck, M Meixner, D Fong, PR McCullough, DE Moser and T Ueta. Large scale extended emission around the Helix Nebula: Dust, molecules, atoms and ions. Astron. J. 2002; 123, 346.

[11] JL Hora, WB Latter, HA Smith and M Marengo. Infrared observations of the Helix planetary nebula. Astrophys. J. 2006; 652, 426-41.

[12] R Bachiller, T Forveille, PJ Huggins and P Cox. The chemical evolution of planetary nebulae. Astron. Astrophys. 1997; 324, 1123-34.

[13] ED Tenenbaum, SN Milam, NJ Woolf and LM Ziurys. Molecular survival in evolved planetary nebulae: Detection of $\mathrm{H}_{2} \mathrm{CO}, \mathrm{c}-\mathrm{C}_{3} \mathrm{H}_{2}$, and $\mathrm{C}_{2} \mathrm{H}$ in the Helix. Astrophys. J. 2009; 704, L108-L112.

[14] LN Zack and LM Ziurys. Chemical complexity in the Helix Nebula: Multi-line observations of $\mathrm{H}_{2} \mathrm{CO} \mathrm{HCO}^{+}$and CO. Astrophys. J. 2013; 765, 112.

[15] M Etxaluze, J Cernicharo, JR Goicoechea, PAMV Hoof, BM Swinyard, MJ Barlow, GCVD Steene, MAT Groenewegen, F Kerschbaum, TL Lim, F Lique, M Matsuura, C Pearson, ET Polehampton, P Royer and T Ueta. Herschel spectral mapping of the Helix Nebula (NGC 7293). Astron. Astrophys. 2014; 566, A78.

[16] DC Martin, J Fanson, D Schiminovich, P Morrissey, PG Friedman, TA Barlow, T Conrow, R Grange, PN Jelinsky, B Milliard, OHW Siegmund, L Bianchi, YI Byun, J Donas, K Forster, TM Heckman, YW Lee, BF Madore, RF Malina, SG Neff, RM Rich, T Small, AS Szalay, TK Wyder. The galaxy evolution explorer: A space ultraviolet survey mission. Astrophys. J. 2005; 619, L1-L6.

[17] L Bianchi, ADL Vega, B Shiao and R Bohlin. New UV-source catalogs, UV spectral database, UV variables and science tools from the GALEX surveys. Astrophys. Space Sci. 2018; 363, 56.

[18] P Morrissey, T Conrow, TA Barlow, T Small, M Seibert, TK Wyder, T Budavári, S Arnouts, PG Friedman and K Forster. The calibration and data products of GALEX. Astrophys. J. Suppl. Ser. $2007 ; 173,682$. 
[19] L Bianchi. The ultraviolet sky surveys: Filling the gap in our view of the universe. Astrophys. Space Sci. 2009; 320, 11-9.

[20] L Bianchi, B Shiao and D Thilker. Revised catalog of GALEX Ultraviolet Sources. I. The all-sky survey: GUVcat_AIS. Astrophys. J. Suppl. 2017; 230, 24.

[21] DT Frayer, D Fadda, L Yan, FR Marleau, PI Choi, G Helou, BT Soifer, PN Appleton, L Armus and R Beck. Spitzer 70 and $160 \mu \mathrm{m}$ observations of the extragalactic first look survey. Astron. J. 2006; 131, 250.

[22] MAT Groenewegen, L Girardi, E Hatziminaoglou, C Benoist, LF Olsen, LD Costa, S Arnouts, R Madejsky, RP Mignani, C Rité, G Sikkema, R Slijkhuis and B Vandame. ESO imaging survey the stellar catalogue in the Chandra deep field south. Astron. Astrophys. 2002; 392, 741-55.

[23] LS Bose, NV Sujatha, K Narayanankutty and J Murthy. Extragalactic survey using GALEX-Spitzer matching fields. Astron. Lett. 2015; 41, 704-11.

[24] S Bowyer. The cosmic far ultraviolet background. Ann. Rev. Astron. Astrophys. 1991; 29, 59-88.

[25] RC Henry. Ultraviolet background radiation. Ann. Rev. Astron. Astrophys. 1991; 29, 89-127.

[26] NV Sujatha, J Murthy, A Karnataki, RC Henry and L Bianchi. GALEX observation of diffuse UV radiation at high spaitial resolution from the Sandage Nebulosity. Astrophys. J. 2009; 692, 1333.

[27] NV Sujatha, J Murthy, R Suresh, RC Henry and L Bianchi. GALEX observations of diffuse ultraviolet emission from Draco. Astrophys. J. 2010; 723, 1549-57.

[28] C Leinert, S Bowyer, LK Haikala, MS Hanner, MG Hauser, AC Levasseur-Regourd, I Mann, K Mattila, WT Reach, W Schlosser, HJ Staude, GN Toller, JL Weiland, JL Weinberg and AN Witt. The 1997 reference of diffuse night sky brightness. Astron. Astrophys. Suppl. Ser. 1998; 127, 1-99.

[29] DJ Schlegel, DP Finkbeiner and M Davis. Maps of dust infrared emission for use in estimation of reddening and cosmic microwave background radiation foregrounds. Astrophys. J. 1998; 500, 525.

[30] LS Bose, NV Sujatha and K Narayanankutty. Ultraviolet and infrared correlation studies in Orion. Open Astron. 2015; 24, 319-26. 